Artículo

\title{
Rendimiento de grano, forraje y calidad forrajera de nuevos híbridos de maíz de Valles Altos
}

\author{
Saúl Velasco Macias ${ }^{1}$ \\ Margarita Tadeo Robledo ${ }^{1 \S}$ \\ Alejandro Espinosa Calderón ${ }^{2}$ \\ Joob Zaragoza Esparza ${ }^{1}$ \\ Enrique Canales Islas ${ }^{3}$ \\ Bulmaro Coutiño Estrada ${ }^{4}$ \\ ${ }^{1}$ Ingeniería Agrícola-Facultad de Estudios Superiores Cuautitlán-Universidad Nacional Autónoma de \\ México. Carretera Cuautitlán-Teoloyucán km 2.5, Cuautitlán Izcalli, Estado de México. CP. 54714. \\ (saulvelasco35@gmail.com; jobzaragoza4920@yahoo.com). ${ }^{2}$ Campo Experimental Valle de México- \\ INIFAP. Carretera Los Reyes-Texcoco km 13.5, Coatlinchan, Texcoco, Estado de México, México. CP. \\ 56250. (espinoale@yahoo.com.mx). $\quad{ }^{3}$ Campo Experimental Santiago Ixcuintla-INIFAP. \\ (enrique1784@yahoo.com.mx). ${ }^{4}$ Campo Experimental Centro de Chiapas-INIFAP. \\ (coutino.bulmaro@inifap.gob.mx). \\ ${ }^{\S}$ Autora para correspondencia: tadeorobledo@yahoo.com.
}

\section{Resumen}

En la Facultad de Estudios Superiores Cuautitlán (FESC-UNAM) y en el Instituto Nacional de Investigaciones Forestales, Agricolas y Pecuarias (INIFAP) se cuentan con híbridos en fase experimental o de reciente liberación para los Valles Altos de México, los cuales son competitivos con los híbridos comerciales ya existentes. En esta investigación se evaluaron 10 híbridos de maíz en dos localidades y en una de ellas con dos fechas de siembra; es decir; tres ambientes diferentes de evaluación en el Estado de México, en cada ambiente se establecieron dos experimentos con los 10 genotipos, en el primero el objetivo fue determinar la productividad de grano y en el segundo fueron determinar la productividad y calidad de forraje. Se utilizó un diseño experimental de bloques completos al azar con 10 genotipos y tres repeticiones, el análisis estadístico se realizó con un arreglo factorial, que consideró los 10 híbridos y tres ambientes, así como la interacción genotipo x ambiente. En conclusión, se determinó que el híbrido Atziri Puma, presentó mayor rendimiento de grano (8 $044 \mathrm{~kg} \mathrm{ha}^{-1}$ ) seguido por Tsíri Puma (7 $783 \mathrm{~kg} \mathrm{ha}^{-1}$ ), ambos recientemente liberados comercialmente por la FES Cuautitlán, UNAM. El híbrido Tlaoli Puma presentó el mayor rendimiento de forraje verde (70 $\left.202 \mathrm{~kg} \mathrm{ha}^{-1}\right)$. En los análisis de calidad de forraje, no se observaron diferencias significativas entre híbridos. Atziri puma, Tlaoli puma y Tsiri puma, ya tienen su registro ante el Catálogo Nacional de Variedades Vegetales (CNVV) y Título de Obtentor.

Palabras clave: Zea mays, calidad de forraje, genotipos, productividad, rendimiento de grano.

Recibido: octubre de 2021

Aceptado: febrero de 2022 


\section{Introducción}

El maíz es el cultivo del cual se obtiene cada año la mayor producción mundial de grano (1 060 millones de toneladas). Existen diferentes variedades de maíz para cada infinidad de agrosistemas, manejo del cultivo, ambientes, altitud, con uso específico y especializado por los agricultores. Se conocen más de 600 diferentes usos. El 76\% del maíz se cultiva bajo humedad de lluvia irregular y escasa y $24 \%$ restante bajo riego. En México, centro de origen, se producen 22.1 millones de toneladas, con un rendimiento medio de $2.8 \mathrm{t} \mathrm{ha}^{-1}$, cada año se recurre a la importación de 16 a 18 millones de toneladas de maíz amarillo, por lo que se requieren en total 32.1 millones de toneladas, consumo total aparente (Espinosa y Tadeo, 2018a; Espinosa et al., 2019). De acuerdo con la SADER (2020), en México se siembran de 7.4 a 7.7 millones de hectáreas desde el año 2010 al 2018 en 2.3 millones de unidades de producción.

Desde la década de los 80's en la Facultad de Estudios Superiores Cuautitlán de la UNAM (FES Cuautitlán, UNAM) y en el Campo Experimental Valle de México del INIFAP (CEVAMEXINIFAP), se generan, desarrollan y evalúan diversos híbridos blancos androestériles de ciclo intermedio para siembras tempranas, algunos de los cuales han expresado buen rendimiento de forraje (Tadeo-Robledo et al., 2016; Espinosa-Calderón y Tadeo-Robledo, 2018 b; EspinosaCalderón et al., 2019; Zaragoza-Esparza et al., 2019). A partir de un grupo sobresaliente de híbridos trilineales, que poseen androesterilidad para facilitar la producción de semillas (Tadeo-Robledo $e t$ $a l, 2018$ b), se han seleccionado algunos que de manera consistente han presentado ventajas en rendimiento en comparación con testigos y que están inscritos en el Catálogo Nacional de Variedades Vegetales (CNVV) para su uso extensivo comercial.

En los primeros ideotipos (llamados arquetipos) de maíz no se discriminaban de acuerdo con su uso. Se aceptaba que el rendimiento y la calidad del ensilado estaban determinados por el rendimiento de grano y el (\%) de grano por encima del resto de los componentes de la planta (Zaragoza-Esparza et al., 2019). Por lo tanto, los fitomejoradores y productores sostuvieron que los mejores maíces para producción de grano eran los más adecuados para ensilado, así el mejor híbrido para grano era considerado como el mejor forrajero. Como consecuencia, la mejora se dirigió al desarrollo de germoplasma e híbridos para producción de grano únicamente.

Por lo general, los híbridos forrajeros, son seleccionados arbitrariamente por su capacidad productora de materia seca, y poco interés se ha puesto en mejorar su calidad nutritiva. (Peña $e t$ al., 2002). Se consideró conveniente definir los materiales más adecuados, que presenten un alto rendimiento de grano y que además expresen buena productividad y calidad de forraje; por ello en el presente trabajo se estableció, como objetivo determinar el rendimiento de grano, materia verde, materia seca, contenido de proteína y digestibilidad de 10 híbridos de grano blanco para Valles Altos de México, así como definir qué híbrido puede ser utilizado para doble propósito, tanto en producción de grano como de forraje.

\section{Materiales y métodos}

El presente trabajo se realizó en dos localidades, en el ciclo primavera-verano 2016. La primera en el Campo Experimental Valle de México (CEVAMEX), perteneciente al INIFAP, ubicado en Santa Lucia de Prías, Municipio de Texcoco, Estado de México a una altitud de $2240 \mathrm{~m}$, la segunda 
localidad fue en la Facultad de Estudios Superiores Cuautitlán (FES-Cuautitlán), Campo 4, de la Universidad Nacional Autónoma de México (UNAM) ubicado en Cuautitlán Izcalli, Estado de México a una altitud de $2274 \mathrm{~m}$. En la primera localidad, se sembraron dos experimentos en dos fechas de siembra, la primera fue el 27 de mayo de 2016 y la segunda el 24 de junio de 2016, en el caso de la segunda localidad, se manejó solamente una fecha de siembra, el 06 de junio de 2016.

Se evaluaron 10 genotipos, cuatro obtenidos en el programa de mejoramiento genético de la FES Cuautitlán, UNAM y seis del INIFAP. Con los 10 materiales se establecieron dos experimentos, el

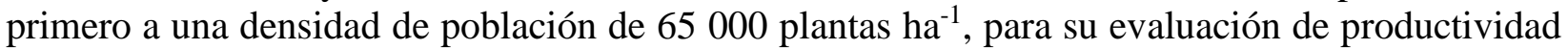
de grano, este experimento se cosechó a los 170 días después de la siembra (Cuadro 1).

Los mismos 10 genotipos, se establecieron en el segundo experimento a una densidad de población de 80000 plantas $\mathrm{ha}^{-1}$, para ser evaluados para producción y calidad de forraje, en este caso la cosecha se efectuó a los 115 días después de la siembra (Cuadro 1). En ambos experimentos se utilizó un diseño experimental bloques completos al azar con 10 genotipos (híbridos), tres repeticiones, cada ensayo se estableció en tres ambientes: dos de ellos en el CEVAMEX (Santa Lucía 1 y Santa Lucía 2) así como otro en la FES Cuautitlán, UNAM. El análisis estadístico se efectuó con arreglo factorial, que consideró a los factores: genotipos (G: 10), ambientes (E: tres), así como la interacción genotipo por ambiente ( $\mathrm{G} \times \mathrm{E})$. Todos los análisis estadísticos se realizaron utilizando el programa SAS versión 9.0 (SAS Institute, 2002).

Cuadro 1. Híbridos de maíz de la UNAM e INIFAP utilizados en dos tipos de experimentos para determinar rendimiento de grano, productividad y calidad de forraje en tres ambientes de evaluación de valles altos en el ciclo primavera-verano 2016.

\begin{tabular}{cccccccc}
\hline $\begin{array}{c}\text { No. } \\
\text { Trat }\end{array}$ & Nombre comercial & $\begin{array}{c}\text { Tipo de } \\
\text { híbrido }\end{array}$ & Origen $\begin{array}{c}\text { Densidad de } \\
\text { plantas (miles) } \\
\text { grano }\end{array}$ & $\begin{array}{c}\text { Días a } \\
\text { cosecha } \\
\text { (grano) }\end{array}$ & $\begin{array}{c}\text { Densidad de } \\
\text { plas (miles) } \\
\text { forraje }\end{array}$ & $\begin{array}{c}\text { Días a } \\
\text { cosecha } \\
\text { (forraje) }\end{array}$ \\
\hline 1 & H 61 & HT & INIFAP & 65 & 170 & 80 & 115 \\
2 & TLAOLI PUMA & HT & UNAM & 65 & 170 & 80 & 115 \\
3 & (IA449RMIA44 X & HCS & UNAM & 65 & 170 & 80 & 115 \\
4 & MIA47) & & & & & & \\
4 & TSIRI PUMA & HT & UNAM & 65 & 170 & 80 & 115 \\
6 & ATZIRI PUMA & HT & UNAM & 65 & 170 & 80 & 115 \\
7 & H 47 AE & HT & INIFAP & 65 & 170 & 80 & 115 \\
8 & H 49 AE & HT & INIFAP & 65 & 170 & 80 & 115 \\
9 & H 53 AE & HT & INIFAP & 65 & 170 & 80 & 115 \\
10 & H 50 & HCD & INIFAP & 65 & 170 & 80 & 115 \\
\hline
\end{tabular}

HT= híbrido trilíneal; HCS= híbrido de cruza simple; $\mathrm{HCD}=$ híbrido de cruza doble.

La parcela experimental estuvo constituida de un surco de $5 \mathrm{~m}$ de largo por $0.8 \mathrm{~m}$ de ancho, dando un total de $4 \mathrm{~m}^{2}$ como parcela útil, con 28 plantas para producción de grano y 32 plantas para producción de forraje. En todos los ambientes, en las tres diferentes fechas de siembra, el terreno se preparó de forma convencional, consistiendo en aradura, dos pasos de rastra y trazo de surcos. Al momento de realizar el surcado se fertilizó con una dosis 80-40-00 (N, P, K), utilizándose urea y fosfato de amonio. 
Se aplicó riego en la siembra y posteriormente se aplicaron dos riegos de auxilio, para el experimento de CEVAMEX, en cambio en la FES Cuautitlán, UNAM, solamente se dio un riego en la siembra y en el resto del ciclo se cubrió el requerimiento hídrico con la humedad de precipitación pluvial. Para el control de maleza se aplicó herbicida a 15 días posteriores al riego, con una mezcla de $3 \mathrm{~kg}$ de Gesaprim calibre 90, 1 L de Hierbamina y 1 L de Sansón 4 SC por hectárea (Tadeo-Robledo et al., 2018). A los 35 días después de la siembra, en cada experimento se ajustó la densidad de población a 32 y 28 plantas por surco para obtener una densidad de 80000 y 65000 plantas $\mathrm{ha}^{-1}$, para los experimentos de forraje y grano, respectivamente.

Para evaluar el rendimiento de grano se evaluaron las variables: rendimiento de grano, días a floración masculina, días a floración femenina, altura de planta, altura de la mazorca, longitud de mazorca, diámetro de mazorca, diámetro de olote, hileras por mazorca, granos por hilera, humedad de grano, peso volumétrico del grano, peso de 200 granos y porcentaje de grano/olote.

Para evaluar el rendimiento de forraje se evaluaron las siguientes variables: rendimiento de materia verde, rendimiento de materia seca, porcentaje de materia seca, porcentaje de mazorca y peso de mazorca seca. La calidad de forraje se evaluó con ayuda de las siguientes variables: porcentaje de proteína y porcentaje de digestibilidad. El porcentaje de proteína se obtuvo mediante el método de Microkjeldahl (AOAC, 2012). En el caso del porcentaje de digestibilidad se determinó mediante el método de digestibilidad in vitro de la materia seca con la técnica de (Tilley y Terry, 1963).

\section{Resultados y discusión}

En los experimentos para determinar productividad de grano, los resultados de las variables analizadas en la evaluación de los 10 híbridos, a través de tres ambientes, mostraron para el factor de variación, ambientes, diferencias altamente significativas $(p<0.01)$, excepto en la altura de mazorca. Para la variable rendimiento de grano, el coeficiente de variación fue de $9.2 \%$ y la media general $6742 \mathrm{~kg} \mathrm{ha}^{-1}$ (Cuadro 2). Para el factor de variación, genotipos (híbridos) se encontraron diferencias altamente significativas en rendimiento y altura de planta. En la interacción ( $\mathrm{G} x \mathrm{E})$ se identificaron diferencias significativas para la variable, altura de planta (Cuadro 2).

Cuadro 2. Cuadrados medios y significancias estadísticas obtenidas de los análisis de varianza para diversas variables para determinar la productividad de grano de diez híbridos de maíz evaluados en tres ambientes de Valles Altos. P-V 2016.

\begin{tabular}{cccccccccc}
\hline $\begin{array}{c}\text { Factor de } \\
\text { variación }\end{array}$ & GL & $\begin{array}{c}\text { Rendimiento grano } \\
\left(\mathrm{kg} \mathrm{ha}^{-1}\right)\end{array}$ & FM & FF & APa & AM & PV & $\%$ MS & P200G \\
\hline AMB & 2 & $6668068.45^{*}$ & $532.07^{* *}$ & $481.23^{* * *}$ & $3390.87^{* *}$ & 23.41 & $39.87^{* *}$ & $71.11^{* *}$ & $1841.41^{* *}$ \\
GEN & 9 & $4988016.13^{* *}$ & 4.19 & 4.19 & $2419.3^{* *}$ & 250.35 & 10 & 0.88 & 88.07 \\
AMB*GEN & 9 & 4598991.49 & 8.22 & 8.22 & $1051.91^{*}$ & 103.02 & 8.91 & 0.96 & 65.34 \\
BLO(AMB) & 9 & 7371237.89 & 1.65 & 1.65 & 1029.02 & 245.45 & 8.5 & 2.15 & 113.34 \\
ERROR & 54 & 1675383 & 3.27 & 3.27 & 515.94 & 160.27 & 6.52 & 0.76 & 46.99 \\
CV & & 19.19 & 2.29 & 2.29 & 9.33 & 10.57 & 3.37 & 1 & 10.26 \\
MEDIA & 6741.84 & 78.7 & 78.7 & 243.31 & 119.71 & 75.77 & 3.08 & 66.81 \\
\hline
\end{tabular}

${ }^{*}=$ significativo al 0.05 de probabilidad de error; ${ }^{* *}=$ altamente significativo al 0.01 de probabilidad de error; FV= factor de variación; $\mathrm{GL}=$ grados de libertad; $\mathrm{FM}=$ floración masculina; $\mathrm{FF}=$ floración femenina; $\mathrm{AP}=$ altura de planta; $\mathrm{AM}=$ altura mazorca; $\mathrm{PV}=$ peso volumétrico; $\mathrm{P} 200 \mathrm{G}=$ peso de 200 granos; $\mathrm{CV}=$ coeficiente de variación . 
En el segundo experimento, para determinar la productividad y calidad de forraje, el análisis de varianza de la evaluación de los 10 híbridos, a través de tres ambientes, detectó para el factor de variación, ambientes, diferencias significativas $(p<0.05)$ para la variable rendimiento de materia verde, así como diferencias altamente significativas $(p<0.01)$ para las variables, rendimiento de materia seca, porcentaje de materia seca, porcentaje de mazorca, peso de mazorca, porcentaje de proteína y porcentaje de digestibilidad (Cuadro 3). Para el factor de variación genotipos, se detectaron diferencias altamente significativas $(p<0.01)$, en las variables rendimiento de materia verde y rendimiento de matera seca. Para las variables porcentaje de mazorca y peso de mazorca, se definieron diferencias significativas $(p<0.05)$ entre los genotipos evaluados, en cambio para las variables $\%$ materia seca, $\%$ proteína y \% digestibilidad no se presentaron diferencias significativas (Cuadro 3).

Para el factor de variación de la interacción (ambiente x genotipo) se encontraron diferencias altamente significativas ( $p<0.01$ ) para las variables: porcentaje de mazorca y peso de mazorca. En el resto de las variables no se detectó significancia estadística (Cuadro 3).

Cuadro 3. Cuadrados medios y significancias estadísticas obtenidas de los análisis de varianza para diversas variables para determinar la productividad y calidad de forraje de diez híbridos de maíz evaluados en tres ambientes de Valles Altos. P-V 2016.

\begin{tabular}{ccccccccc}
\hline $\begin{array}{c}\text { Factor de } \\
\text { variación }\end{array}$ & GL & RMV & RMS & $\% \mathrm{MS}$ & $\% \mathrm{Maz}$ & $\mathrm{PM}$ & $\%$ Prot & \%Digest \\
\hline AMB & 2 & $634092361^{*}$ & $125885209.5^{* *}$ & $315.96^{* *}$ & $6968.35^{* *}$ & $1.073^{* *}$ & $12.68^{* *}$ & $278.71^{* *}$ \\
GEN & 9 & $1436999691^{* *}$ & $106031022.7^{* *}$ & 11.57 & $54.51^{*}$ & $0.023^{*}$ & 1.16 & 19.71 \\
$\mathrm{AMB} * \mathrm{GEN}$ & 9 & 125457330 & 13398110.2 & 16.72 & $55.29^{* *}$ & $0.023^{* *}$ & 1.01 & 17.38 \\
$\mathrm{BLO}(\mathrm{AMB})$ & 9 & 88360417 & 14353318.1 & 37.09 & 24.71 & 0.009 & 2.56 & 45.8 \\
ERROR & 54 & 136618904 & 14805911 & 24.31 & 23.1 & 0.009 & 0.63 & 34.48 \\
CV & & 19.69 & 25 & 18.73 & 16.58 & 21.33 & 8.77 & 8.56 \\
MEDIA & 59355.56 & 15382.19 & 26.31 & 28.97 & & 9.08 & 68.54 \\
\hline
\end{tabular}

${ }^{*}=$ significativo al 0.05 de probabilidad de error; ${ }^{* *}=$ altamente significativo al 0.01 de probabilidad de error; $\mathrm{FV}=$ factor de variación; $\mathrm{GL}=$ grados de libertad; $\mathrm{RMV}=$ rendimiento materia verde; $\mathrm{RMS}=$ rendimiento materia seca; $\% \mathrm{Maz}=$ porcentaje de mazorca; $\mathrm{PM}=$ peso de mazorca; \%Prot= porcentaje proteína; \%Digest= porcentaje digestibilidad; $\mathrm{CV}=$ coeficiente de variación.

Para el primer experimento; es decir, para determinar productividad de grano, en la prueba de comparación de medias en los tres ambientes evaluados (Cuadro 4), en la variable de rendimiento de grano se definieron dos grupos de significancia, ubicándose Santa Lucía 1, primera fecha de siembra, con el mayor rendimiento de grano (7 $\left.282 \mathrm{~kg} \mathrm{ha}^{-1}\right)$, superior en $11.2 \%$ a los rendimientos obtenidos en FES Cuautitlán, UNAM y Santa Lucía, segunda fecha (6 $467 \mathrm{~kg} \mathrm{ha}^{-1}$ ). Dichos resultados son similares a los reportados por Espinosa-Calderón et al. (2018), para la localidad de Santa Lucía (CEVAMEX).

Estos resultados fueron probablemente consecuencia de los 10 días de diferencia en la floración que se tuvo a comparación de FES Cuautitlán, UNAM, lo que provocó posiblemente un mayor aprovechamiento del periodo húmedo en la localidad de Santa Lucía, posiblemente los 18 días de diferencia en la siembra entre la primera fecha y segunda fecha de la localidad de Santa Lucía, 
también sea la consecuencia de la diferencia de rendimiento entre ambos ambientes; así también, en Santa Lucía se aplicaron riegos de auxilio a comparación con FES Cuautitlán, UNAM, en donde se cubrieron los requerimientos hídricos del cultivo con la precipitación.

En la variable altura de planta, el ambiente Santa Lucia 1, fue superior y diferente significativamente a los otros dos ambientes, lo que probablemente influyó en el rendimiento de grano y forraje (Zaragoza-Esparza et al., 2019). Para la variable porcentaje de grano se definieron tres grupos de significancia; es decir, se obtuvieron diferencias significativas en las tres localidades, en cambio, en las variables peso volumétrico y peso de 200 granos, se definieron dos grupos de significancia, donde los valores altos se obtuvieron en FES Cuautitlán, UNAM, en comparación con los ambientes de Santa Lucía. Esto pudo ser consecuencia de un ambiente que favoreció el crecimiento y desarrollo del cultivo y por lo tanto, la formación de mazorca (Cuadro 4).

Cuadro 4. Comparación de medias entre ambientes para diversas variables para determinar la productividad de grano considerando la media de diez híbridos de maíz. P-V 2016.

\begin{tabular}{cccccccccc}
\hline Ambiente & $\begin{array}{c}\text { Rendimiento de } \\
\text { grano }\left(\mathrm{kg} \mathrm{ha}^{-1}\right)\end{array}$ & $\begin{array}{c}\text { FM } \\
(\text { días })\end{array}$ & $\begin{array}{c}\text { FF } \\
(\text { días })\end{array}$ & $\begin{array}{c}\text { AP } \\
(\mathrm{cm})\end{array}$ & $\begin{array}{c}\text { AM } \\
(\mathrm{cm})\end{array}$ & $\% \mathrm{MS} \% \mathrm{GV}$ & PV $\left(\mathrm{kg} \mathrm{hL}^{-1}\right)$ & $\begin{array}{c}\mathrm{P} 200 \mathrm{G} \\
(\mathrm{g})\end{array}$ \\
\hline Santa Lucía 1 & $7282 \mathrm{a}$ & $75 \mathrm{~b}$ & $78 \mathrm{~b}$ & $255 \mathrm{a}$ & $119 \mathrm{a}$ & $88 \mathrm{a}$ & $85 \mathrm{~b}$ & $75 \mathrm{ab}$ & $61.4 \mathrm{~b}$ \\
FESC UNAM & $6467 \mathrm{~b}$ & $82 \mathrm{a}$ & $83 \mathrm{a}$ & $238 \mathrm{~b}$ & $121 \mathrm{a}$ & $85 \mathrm{~b}$ & $87 \mathrm{a}$ & $77 \mathrm{a}$ & $75.8 \mathrm{a}$ \\
Santa Lucía 2 & $6467 \mathrm{~b}$ & $74 \mathrm{~b}$ & $75 \mathrm{c}$ & $236 \mathrm{~b}$ & $120 \mathrm{a}$ & $88 \mathrm{a}$ & $83 \mathrm{c}$ & $75 \mathrm{~b}$ & $63.2 \mathrm{~b}$ \\
DSH (0.05) & 805 & 1 & 1 & 14 & 8 & 0.54 & 0.97 & 2 & 4.26 \\
\hline
\end{tabular}

$\mathrm{DSH}=$ diferencia significativa honesta $(p<0.05) ; \mathrm{FM}=$ floración masculina; $\mathrm{FF}=$ floración femenina; $\mathrm{AP}=$ altura de planta; $\mathrm{AM}=$ altura mazorca; $\% \mathrm{MS}=$ porcentaje de materia seca; $\% \mathrm{G}=$ porcentaje de grano; $\mathrm{PV}=$ peso volumétrico; $\mathrm{P} 200 \mathrm{G}=$ peso de 200 granos.

Para el segundo experimento; es decir, para determinar productividad y calidad de forraje, en la prueba de comparación de medias en los tres ambientes evaluados (Cuadro 5), en la variable para rendimiento de materia verde se definieron dos grupos de significancia, ubicándose Santa Lucía 1 (primera fecha de siembra), con el mayor rendimiento de forraje verde (64 $392 \mathrm{~kg} \mathrm{ha}^{-1}$ ), que fue 9.5\% superior en comparación al rendimiento de Santa Lucia 2 (segunda fecha) y éste a su vez, $14 \%$ más en comparación a FES Cuautitlán, UNAM (55 $\left.383 \mathrm{~kg} \mathrm{ha}^{-1}\right)$. Estos resultados son superiores a los reportados por Elizondo y Boschino (2001); Hernández-Sánchez et al. (2011).

Se puede considerar que el porcentaje de mazorcas fue la variable que incidió directamente al rendimiento de materia verde, esto se debe a que cuando aumenta la densidad de población (de 65 000 a 80000 plantas ha $^{-1}$ ) aumenta la intercepción de la radiación fotosintética activa por el dosel, promoviendo así la acumulación de materia seca a nivel de población, especialmente para las variedades tempranas (Li et al., 2020), en este sentido, la primera fecha de siembra de los genotipos, con una diferencia de 28 días con respecto a la segunda fecha en la primera localidad y 10 días con respecto a la segunda, repercutieron directamente en el rendimiento de forraje.

Para la comparación de medias en la variable rendimiento de materia seca, se definieron dos grupos de significancia, se tuvo el mayor rendimiento (17 $458.3 \mathrm{~kg} \mathrm{ha}^{-1}$ ) en Santa Lucía 2 (segunda fecha), esto se puede deber a la menor cantidad de humedad, debido a las bajas precipitaciones en comparación a FES Cuautitlán, UNAM y a la siembra tardía a comparación de la primera fecha. El rendimiento de materia seca está relacionado con el porcentaje de materia seca, por lo que los resultados en dichas variables son similares (Cuadro 5). 
Cuadro 5. Comparación de medias entre ambientes para diversas variables para determinar la productividad y calidad de forraje considerando la media de 10 híbridos de maíz. PV 2016.

\begin{tabular}{cccccccc}
\hline Ambiente & RMV & RMS & $\%$ MS & $\%$ Maz & PM & $\%$ Prot & \%Digest \\
\hline FESC UNAM & $55383 \mathrm{~b}$ & $13362 \mathrm{~b}$ & $24.3 \mathrm{~b}$ & $15.8 \mathrm{c}$ & $0.6 \mathrm{a}$ & $8.58 \mathrm{~b}$ & $69.56 \mathrm{a}$ \\
Santa Lucía 1 & $64392 \mathrm{a}$ & $15326 \mathrm{ab}$ & $24.5 \mathrm{~b}$ & $45.7 \mathrm{a}$ & $0.3 \mathrm{c}$ & $8.84 \mathrm{~b}$ & $65.13 \mathrm{~b}$ \\
Santa Lucía 2 & $58290 \mathrm{ab}$ & $17458 \mathrm{a}$ & $30 \mathrm{a}$ & $25.4 \mathrm{~b}$ & $0.5 \mathrm{~b}$ & $9.82 \mathrm{a}$ & $70.94 \mathrm{a}$ \\
DSH (0.05) & 7273 & 2394 & 3.1 & 2.99 & 0.061 & 0.49 & 3.65 \\
\hline
\end{tabular}

$\mathrm{DSH}=$ diferencia significativa honesta $(p<0.05) ; \mathrm{RMV}=$ rendimiento materia verde; $\mathrm{RMS}=$ rendimiento materia seca; $\% \mathrm{Maz}=$ porcentaje de mazorca; $\mathrm{PM}=$ peso de mazorca; \%Prot= porcentaje proteína; \%Digest= porcentaje digestibilidad.

Para la variable porcentaje de proteína se definieron dos grupos de significancia, ubicándose en el valor más alto el ambiente de Santa Lucía 2 (segunda fecha), el cual tuvo 9.82\% de proteína. Los otros dos ambientes no mostraron diferencias significativas (Cuadro 5). Otra variable importante para la calidad de forraje es el porcentaje de digestibilidad en donde no se encontraron diferencias significativas entre FES Cuautitlán, UNAM (69.56\%) y Santa Lucía segunda fecha (70.94\%).

En la comparación de medias para los 10 genotipos evaluados, en el primer tipo de experimentos, (determinación de productividad de grano), considerando la media de los tres ambientes de evaluación, en la variable rendimiento de grano se definieron dos grupos de significancia, ubicándose al híbrido Atziri Puma con el mayor rendimiento de grano, al 12\% de humedad (8044 $\mathrm{kg} \mathrm{ha}^{-1}$ ), superior estadísticamente al híbrido H 51 AE y al H 47 AE. Dichos resultados son similares a los reportados en otros trabajos de investigación (Tadeo et al., 2016; Tadeo et al., 2018a; Tadeo et al., 2018 b; Tadeo-Robledo et al., 2020).

Los resultados obtenidos se encuentran dentro del rango de rendimiento de grano (3.89 a $11.71 \mathrm{~kg}$ $\mathrm{ha}^{-1}$ ) reportado por Velasco-García et al. (2019), para Valles altos de los trópicos mexicanos. En CEVAMEX, el híbrido Atziri Puma exhibe rendimientos por arriba de lo obtenido en los estudios de (Tadeo et al., 2018a; Tadeo et al., 2018b; Tadeo-Robledo et al., 2020) que mostraron rendimiento de que van de 6876 hasta $7528 \mathrm{~kg} \mathrm{ha}^{-1}$.

El segundo híbrido con mayor rendimiento de grano fue Tsíri Puma con $7783 \mathrm{~kg} \mathrm{ha}^{-1}$, lo que es importante, ya que este híbrido fue recientemente liberado para su uso de manera comercial, está inscrito en el Catálogo Nacional de Variedades Vegetales (CNVV), tiene título de obtentor (TadeoRobledo et al., 2016). Los otros genotipos exhibieron rendimientos similares estadísticamente. Los híbridos Tsíri Puma, H49AE y H51AE presentaron plantas con mayor altura. En las variables floración masculina, floración femenina y altura de mazorca, no se presentaron diferencias significativas (Cuadro 6).

En la comparación de medias para las variables, porcentaje de materia seca, porcentaje de grano y peso volumétrico no se presentan diferencias significativas entre los genotipos evaluados. Para el peso de 200 granos se observaron diferencias entre el híbrido H 51 AE que fue superior al H 62 (Cuadro 6). 
Cuadro 6. Comparación de medias entre ambientes para diversas variables para determinar la productividad y calidad de forraje considerando la media de diez híbridos de maíz. PV 2016.

\begin{tabular}{cccccccccc}
\hline \multirow{2}{*}{ Genotipo } & $\begin{array}{c}\text { Rendimiento } \\
\text { de grano }\end{array}$ & FM & FF & AP & AM & MS & G & PV & P200G \\
\cline { 2 - 9 } & $\left(\mathrm{kg} \mathrm{ha}^{-1}\right)$ & (días) & $($ días $)$ & $(\mathrm{cm})$ & $(\mathrm{cm})$ & $(\%)$ & $(\%)$ & $\left(\mathrm{kg} \mathrm{hl}^{-1}\right)$ & $(\mathrm{g})$ \\
\hline Atziri Puma & $8044 \mathrm{a}$ & $77 \mathrm{a}$ & $80 \mathrm{a}$ & $241 \mathrm{abc}$ & $120 \mathrm{a}$ & $86 \mathrm{a}$ & $86 \mathrm{a}$ & $75 \mathrm{a}$ & $66 \mathrm{ab}$ \\
Tsíri Puma & $7783 \mathrm{ab}$ & $77 \mathrm{a}$ & $78 \mathrm{a}$ & $268 \mathrm{a}$ & $111 \mathrm{a}$ & $87 \mathrm{a}$ & $87 \mathrm{a}$ & $77 \mathrm{a}$ & $65 \mathrm{ab}$ \\
H 49AE & $7002 \mathrm{ab}$ & $77 \mathrm{a}$ & $79 \mathrm{a}$ & $256 \mathrm{ab}$ & $114 \mathrm{a}$ & $87 \mathrm{a}$ & $87 \mathrm{a}$ & $77 \mathrm{a}$ & $69 \mathrm{ab}$ \\
(ENS4@ QRL-\#) X MIA47 & $6969 \mathrm{ab}$ & $77 \mathrm{a}$ & $80 \mathrm{a}$ & $242 \mathrm{ab}$ & $122 \mathrm{a} 87 \mathrm{a}$ & $87 \mathrm{a}$ & $76 \mathrm{a}$ & $66 \mathrm{ab}$ \\
H 53AE & $6752 \mathrm{ab}$ & $77 \mathrm{a}$ & $79 \mathrm{a}$ & $256 \mathrm{ab}$ & $120 \mathrm{a}$ & $86 \mathrm{a}$ & $86 \mathrm{a}$ & $74 \mathrm{a}$ & $67 \mathrm{ab}$ \\
Tlaoli Puma & $6689 \mathrm{ab}$ & $77 \mathrm{a}$ & $78 \mathrm{a}$ & $230 \mathrm{bc}$ & $118 \mathrm{a} 87 \mathrm{a}$ & $87 \mathrm{a}$ & $76 \mathrm{a}$ & $70 \mathrm{ab}$ \\
H-61 & $6334 \mathrm{ab}$ & $76 \mathrm{a}$ & $77 \mathrm{a}$ & $233 \mathrm{abc}$ & $119 \mathrm{a} 87 \mathrm{a}$ & $87 \mathrm{a}$ & $74 \mathrm{a}$ & $62 \mathrm{~b}$ \\
H-50 & $6049 \mathrm{ab}$ & $77 \mathrm{a}$ & $79 \mathrm{a}$ & $237 \mathrm{abc}$ & $124 \mathrm{a} 87 \mathrm{a}$ & $87 \mathrm{a}$ & $76 \mathrm{a}$ & $66 \mathrm{ab}$ \\
H 51AE & $5986 \mathrm{~b}$ & $76 \mathrm{a}$ & $78 \mathrm{a}$ & $256 \mathrm{ab}$ & $131 \mathrm{a} 87 \mathrm{a}$ & $87 \mathrm{a}$ & $77 \mathrm{a}$ & $72 \mathrm{a}$ \\
H 47AE & $5811 \mathrm{~b}$ & $77 \mathrm{a}$ & $78 \mathrm{a}$ & 212 & $118 \mathrm{a} 87 \mathrm{a}$ & $87 \mathrm{a}$ & $77 \mathrm{a}$ & $63 \mathrm{ab}$ \\
DSH 0.05 & 2013 & 3 & 3 & 35 & 20 & 1 & 1 & 4 & 10.66 \\
\hline
\end{tabular}

$\mathrm{DSH}=$ diferencia significativa honesta $(p<0.05) ; \mathrm{FM}=$ floración masculina; $\mathrm{FF}=$ floración femenina; $\mathrm{AP}=$ altura de planta; $\mathrm{AM}=$ altura mazorca; $\% \mathrm{MS}=$ porcentaje de materia seca; $\% \mathrm{G}=$ porcentaje de grano; $\mathrm{PV}=$ peso volumétrico; $\mathrm{P} 200 \mathrm{G}=$ peso de 200 granos.

Para el segundo tipo de experimento, de productividad y calidad de forraje, en la prueba de comparación de medias entre los 10 híbridos para los tres ambientes evaluados, en la variable rendimiento de materia verde. Se detectaron cuatro grupos de significancia, ubicándose al híbrido Tlaoli Puma con el mayor rendimiento de forraje verde (70 $\left.202 \mathrm{~kg} \mathrm{ha}^{-1}\right)$, superior estadísticamente a H 51 AE y H 47 AE.

Por su parte, el híbrido H 47AE presentó el menor rendimiento en comparación con los demás híbridos, siendo inferior en $46.4 \%$ al híbrido Tlaoli Puma, lo anterior es importante ya que Tlaoli Puma fue registrado ante el Catálogo Nacional de Variedades Vegetales (CNVV) y cuenta con título de obtentor a nombre de la UNAM (Tadeo-Robledo et al., 2018; Tadeo-Robledo et al., 2020). En segundo lugar, se ubicó el híbrido Tsíri Puma con $68750 \mathrm{~kg} \mathrm{ha}^{-1}$. El híbrido Tlaoli Puma tuvo un rendimiento medio de materia seca de 19.6 ton ha ${ }^{-1}$, siendo superior $(p<0.05)$ a los híbridos $\mathrm{H}$ $61, \mathrm{H} 51 \mathrm{AE}$ y $\mathrm{H} 47 \mathrm{AE}$ en $34.1 \%, 37 \%$ y $59 \%$, respectivamente. En peso de mazorca, el híbrido H 53AE fue superior ( $p<0.05)$ a los híbridos H 61 y H 47AE (Cuadro 7).

Los tres mejores materiales en rendimiento de materia seca fueron Tlaoli Puma, Tsíri Puma y H 49 $\mathrm{AE}$, con $19615 \mathrm{~kg} \mathrm{ha}^{-1}, 17567 \mathrm{~kg} \mathrm{ha}^{-1}$ y $17474 \mathrm{~kg} \mathrm{ha}^{-1}$ respectivamente, los tres se ubican en el mismo grupo de significancia y superan al híbrido H 50 (Cuadro 7). Este híbrido en particular exhibió un rendimiento de materia seca de $15278 \mathrm{~kg} \mathrm{ha}^{-1}$, lo cual indica que los materiales señalados con mejores rendimientos podrían difundirse para su uso extensivo, que bien podrían sustituir al H 50, que es el maíz más sembrado en los Valles Altos de México, desde hace 22 años (Espinosa-Calderón et al., 2019). 
En la comparación de medias de los 10 genotipos para las variables de porcentaje de proteína y porcentaje de digestibilidad que representan la calidad de forraje no mostraron diferencias significativas, lo cual es un buen indicador de la calidad forrajera que tiene cada uno de ellos, ya que todos se ubicaron dentro de valores aceptables para dichas variables (Cuadro 7). Bajo condiciones áridas como la Región Lagunera, Coahuila, utilizando la misma densidad de población que se usó en esta investigación ( 80000 plantas ha $^{-1}$ ) e incrementando el riego conforme aumentaba la evapotranspiración (de 60 al 100\%), se obtuvieron rendimientos desde $47665 \mathrm{~kg} \mathrm{ha}^{-1}$ hasta 57 $665 \mathrm{~kg} \mathrm{ha}^{-1}$ (Yescas et al., 2015), lo que evidencia que la cantidad de agua suministrada al cultivo provoca una suficiencia o carencia de humedad al suelo, lo cual se refleja en el rendimiento del cultivo y en sus diferentes variables como forraje en verde y rendimiento de materia seca.

Cuadro 7. Comparación de medias entre híbridos para determinar la productividad y calidad de forraje considerando la media de tres ambientes de evaluación en Valles Altos. P-V 2016.

\begin{tabular}{cccccccc}
\hline Genotipo & RMV & RMS & \%MS & \%Maz & PM & \%Prot & \%Digest \\
\hline Tlaoli Puma & $70202 \mathrm{a}$ & $19615 \mathrm{a}$ & $27 \mathrm{a}$ & $27 \mathrm{a}$ & $0.5 \mathrm{ab}$ & $9.7 \mathrm{a}$ & $67 \mathrm{a}$ \\
Tsíri Puma & $68750 \mathrm{ab}$ & $17567 \mathrm{abc}$ & $26 \mathrm{a}$ & $31 \mathrm{a}$ & $0.5 \mathrm{ab}$ & $9.6 \mathrm{a}$ & $67 \mathrm{a}$ \\
H 49AE & $68472 \mathrm{ab}$ & $17474 \mathrm{abc}$ & $25 \mathrm{a}$ & $28 \mathrm{a}$ & $0.5 \mathrm{ab}$ & $9.3 \mathrm{a}$ & $68.5 \mathrm{a}$ \\
H 53AE & $66694 \mathrm{ab}$ & $15876 \mathrm{abc}$ & $24 \mathrm{a}$ & $27 \mathrm{a}$ & $0.6 \mathrm{a}$ & $9.3 \mathrm{a}$ & $70.3 \mathrm{a}$ \\
Atziri Puma & $66333 \mathrm{ab}$ & $18543 \mathrm{ab}$ & $28 \mathrm{a}$ & $29 \mathrm{a}$ & $0.4 \mathrm{ab}$ & $9.2 \mathrm{a}$ & $68.3 \mathrm{a}$ \\
(ENS4@QRL\#) x MIA47 & $63417 \mathrm{ab}$ & $16055 \mathrm{abc}$ & $25 \mathrm{a}$ & $26 \mathrm{a}$ & $0.5 \mathrm{ab}$ & $9.0 \mathrm{a}$ & $70 \mathrm{a}$ \\
H 50 & $58944 \mathrm{abc}$ & $15278 \mathrm{abc}$ & $26 \mathrm{a}$ & $27 \mathrm{a}$ & $0.4 \mathrm{ab}$ & $9.0 \mathrm{a}$ & $69 \mathrm{a}$ \\
H 61 & $51167 \mathrm{bc}$ & $12922 \mathrm{bcd}$ & $26 \mathrm{a}$ & $30 \mathrm{a}$ & $0.4 \mathrm{~b}$ & $8.7 \mathrm{a}$ & $68.1 \mathrm{a}$ \\
H 51AE & $45194 \mathrm{~cd}$ & $12419 \mathrm{~cd}$ & $26 \mathrm{a}$ & $32 \mathrm{a}$ & $0.4 \mathrm{ab}$ & $8.7 \mathrm{a}$ & $71 \mathrm{a}$ \\
H 47AE & $32556 \mathrm{~d}$ & $8072 \mathrm{~d}$ & $27 \mathrm{a}$ & $33 \mathrm{a}$ & $0.3 \mathrm{~b}$ & $8.7 \mathrm{a}$ & $67 \mathrm{a}$ \\
DSH (0.05) & 18179 & 5984.4 & 7.66 & 7.47 & 0.15 & 1.23 & 9.13 \\
\hline
\end{tabular}

$\mathrm{DSH}=$ diferencia significativa honesta $(p<0.05) ; \mathrm{RMS}=$ rendimiento materia seca; \% Maz= porcentaje de mazorca; $\mathrm{PM}=$ peso de mazorca; \%Prot= porcentaje proteína; \%Digest= porcentaje digestibilidad.

La media obtenida para porcentaje de proteína fue de $9.12 \%$, cifra superior a lo reportado por Núñez-Hernández et al. (2015), quién además obtuvo una media de porcentaje de digestibilidad de 8.6\%. Yescas et al. (2015) reportaron que ante un aumento de la lámina de riego y la densidad de población (de 80000 a 160000 plantas $\mathrm{ha}^{-1}$ ), el porcentaje de proteína no aumenta significativamente, encontrando valores de 8.51 a $8.6 \%$ bajo una densidad de 80000 plantas ha $^{-1}$, caso similar con \% expresado por los híbridos evaluados en esta investigación (de 8.7 a 9.7\%); sin embargo, cabe resaltar que en dicha investigación solo se evaluó un híbrido. Las diferencias aquí encontradas pueden atribuirse a la constitución genética que tiene cada híbrido evaluado (ArellanoVázquez et al., 2017), recordando que éstos son cruzas simples, cruzas trilineales y cruzas dobles.

En la comparación de medias entre genotipos no se encuentran diferencias significativas $(p<0.05)$. Se obtuvo una media de $68.2 \%$ de digestibilidad in vitro para los híbridos evaluados. Para la comparación de medias entre los híbridos no se presentan diferencias significativas $(p<0.05)$ pero el híbrido de cruza simple (ENS4@QRL-\#) xMIA47 presentó el valor más alto de esta característica. 
Dichos resultados son similares a los obtenidos por Núñez-Hernández et al. (2015) quiénes reportan que los valores de digestibilidad in vitro varían de 62.6 a $67.8 \%$ en los híbridos de ciclo intermedio y de 67.2 a $73.2 \%$ en los híbridos precoces.

\section{Conclusiones}

El híbrido Atziri Puma, presentó el mayor rendimiento de grano, que fue superior estadísticamente a los híbridos H 51 AE y H 47 AE. El híbrido Tlaoli Puma exhibió el mayor rendimiento de forraje verde, superior estadísticamente a $\mathrm{H} 51$ AE y H 47 AE. Con base en los rendimientos de grano, forraje y su calidad, los híbridos Atziri Puma y Tlaoli Puma, mostraron características favorables para considerarse materiales de doble propósito.

\section{Agradecimientos}

El presente trabajo se llevó a cabo con financiamiento del Programa de Apoyo a Proyectos de Investigación e Innovación Tecnológica (PAPIIT), de la Dirección General del Personal Académico de la UNAM. Clave: IT201618.

\section{Literatura citada}

AOAC. 2012. Official methods of analysis of AOAC International 19 (Ed.). Vol. 1. Washington, DC. USA. 672 p.

Elizondo, J. y Boschino, C. 2001. Efecto de la densidad de siembra sobre el rendimiento y calidad del forraje de maíz. Agronomía Mesoamericana. 12(2):181-187.

Espinosa-Calderón A. y Tadeo-Robledo, M. 2018. Importación récord de maíz, una afrenta para México. In: Economía. La Jornada. https://www.jornada.com.mx/2018/02/25/economia/ 021a1eco.

Espinosa-Calderón, A.; Tadeo-Robledo, M. y Zamudio- González, B. 2018 . H-47 AE, Híbrido de maíz para Valles Altos de México. Rev. Fitotec. Mex. 41(1):87-88.

Espinosa-Calderón, A.; Tadeo-Robledo, M.; Turrent-Fernández, A.; Gómez-Montiel, N.; SierraMacías, N.; Palafox-Caballero, A.; Caballero-Hernández, F.; Valdivia-Bernal, R. y Rodríguez-Montalvo. 2008. El potencial de las variedades nativas y mejoradas de maíz. Ciencias. 92(93):118-125.

Espinosa-Calderón, A.; Tadeo-Robledo, M.; Virgen, V. J.; Zamudio-González, B.; TurrentFernández, A.; López-López, C. 2019. H 47AE, H 49AE y H 53AE híbridos de maíz con androesterilidad para Valles Altos. Primer simposio para la autosuficiencia y soberanía alimentaria de México. V reunión estatal de investigación. Publicación especial núm. 1, Campo Experimental Valle de México, INIFAP. Texcoco, Estado de México. 8-12 pp.

Li, J.; Wu, M.; Wang, K.; Ming, B.; Chang, X.; Wang, X.; Ynag, Z.; Xie, R. and Li, S. 2020. Identifying ways to narrow maize yield gaps based on plant density experiments. Agronomy. 10(2):281-293.

Núñez-Hernández, G.; Anaya-Salgado, A.; Faz-Contreras, R. y Serrato-Medina, H. 2015. Híbridos de maíz forrajero con alto potencial de producción de leche de bovino. Agrofaz. 15(1):49-56. 
Peña-Ramos, A.; Núñez-Hernández, G. y González-Castañeda, F. 2002. Potencial forrajero de poblaciones de maíz y relación entre atributos agronómicos con la calidad. Téc. Pecu. Méx. 40(3):215-228.

SAS, Institute. 2002. Statistical Analysis System User's Guide. SAS Institute. Cary. USA. 956.

SADER. 2020. Secretaría de Agricultura y Desarrollo Rural Reporte del mercado de maíz.:18. https://www.cima.aserca.gob.mx/work/models/cima/pdf/cadena/2020/Reporte_mercado_ maiz_2 00120.pdf.

Sánchez-Hernández, M.; Aguilar-Martínez, C.; Valenzuela-Jiménez, N.; Sánchez-Hernández, C.; Jiménez-Rojas, M. y Villanueva-Verduzco, C. 2011. Densidad de siembra y crecimiento de maíces forrajeros. Agronomía Mesoamericana. 22(2):281-295. http://www.scielo.sa.cr/ scielo.php?script=sci_arttext\&pid=S1659-13212011000200005 \&lng=en\&tlng=es.

Tadeo-Robledo, M.; Espinosa-Calderón, A.; García-Zavala, J. J.; Lobato-Ortiz, R.; GómezMontiel, N. O.; Sierra-Macías, M.; Valdivia-Bernal, R.; Zamudio-González, B.; MartínezYáñez, B.; López-López, C.; Mora-García, K. Y.; Canales-Islas, E. I.; Cárdenas-Marcelo, A. L.; Zaragoza-Esparza, J. y Alcántar-Lugo, H. J. 2016. Tsíri puma, híbrido de maíz para Valles Altos con esquema de Androesterilidad para producción de semillas. Rev. Fitotec. Mex. 39(3):331-333.

Tadeo-Robledo, M.; Espinosa-Calderón, A.; García-Zavala, J. J.; Lobato-Ortiz, R.; GómezMontiel, N. O.; Sierra-Macías, M.; Valdivia-Bernal, R.; Turrent-Fernández, A. and Zamudio-González, B. 2018 b. Productivity of three maize hybrids under different proportions of male sterile and fertile seeds. Interciencia. 43(12):852-857.

Tadeo-Robledo, M.; Espinosa-Calderón, A.; López-López, C.; Canales-Islas, E. I.; ZaragozaEsparza, J.; Sierra-Molina, M. y Gómez-Montiel, N. 2018 a. Atziri Puma y Tlaoli Puma nuevos híbridos de maíz blanco con restauración de la fertilidad masculina en la producción de semilla. Acta Fitogenetica. 5(1):85-85.

Tadeo-Robledo, M.; Espinosa-Calderón, A.; Canales-Islas, R. I.; López-López, C.; ZamudioGonzález, B.; Turrent-Fernández, A.; Gómez-Montiel, N.; Sierra-Macías, M.; MartínezGutiérrez, A.; Valdivia-Bernal. and Andrés-Meza, P. 2020. Grain yield and population densities of new corn hybrids released by the INIFAP and UNAM for the High Valleys of Mexico. Terra Latinoam. 38(3):507-515.

Tilley, J. M. A. and Terry, R. A. 1963. A two-stage technique for the in vitro digestion of forage crops. J. Br. Grassland Soc. 18:104-111.

Van Soest P. J. 1967. Development of a comprehensive system of feed analices and its application to forages. J. Anim. Sci. 26(18):119-128.

Velasco-García, J.; García-Zavala, J.; Sahagún-Castellanos, J.; Lobato-Ortiz, R.; Sánchez-Abarca, C. y Marín-Montes, I. M. 2019. Rendimiento, componentes de rendimiento y heterosis de germoplasma de maíz adaptado a Valles Altos. Rev. Fitotec. Mex. 42(4):367-374.

Yescas, C. P.; Segura, C. M.; Martínez, C. L.; Álvarez, R. V.; Montemayor, T. J.; Orozco, V. J. y Frías, R. J. 2015. Rendimiento y calidad de maíz forrajero (Zea mays L.) con diferentes niveles de riego por goteo subsuperficial y densidad de plantas. Pyton. 84(2):272-279.

Zaragoza-Esparza, J.; Tadeo-Robledo, M.; Espinosa-Calderón, A.; López-López, C.; GarcíaEspinosa, J. C.; Zamudio-González, B.; Turrent-Fernández, A. y Rosado-Núñez, F. 2019. Rendimiento y calidad de forraje de híbridos de maíz en Valles Altos de México. Rev. Mex. Cienc. Agríc. 10(1):101-115. 